\title{
Miranda
}

Revue pluridisciplinaire du monde anglophone /

Multidisciplinary peer-reviewed journal on the English-

speaking world

15 | 2017

Lolita at 60 / Staging American Bodies

\section{Nabokov et la censure}

Julie Loison-Charles

\section{CpenEdition}

Journals

Édition électronique

URL : http://journals.openedition.org/miranda/11223

DOI : 10.4000/miranda. 11223

ISSN : 2108-6559

Éditeur

Université Toulouse - Jean Jaurès

Référence électronique

Julie Loison-Charles, « Nabokov et la censure », Miranda [En ligne], 15 | 2017, mis en ligne le 06 octobre 2017, consulté le 16 février 2021. URL : http://journals.openedition.org/miranda/11223 ; DOI : https:// doi.org/10.4000/miranda.11223

Ce document a été généré automatiquement le 16 février 2021.

\section{c) () $\Theta$}

Miranda is licensed under a Creative Commons Attribution-NonCommercial-NoDerivatives 4.0 International License. 


\title{
Nabokov et la censure
}

\author{
Julie Loison-Charles
}

1 Le roman le plus connu de Nabokov reste Lolita, comme en atteste la trace que ce livre a laissée dans la culture populaire (héritage visuel dans la culture manga et la mode féminine, références dans des chansons françaises ou anglo-saxonnes) et plus généralement dans la langue : une lolita, avec sa perte de majuscule symptomatique de l'antonomase, est maintenant perçue principalement comme une jeune fille à la sexualité précoce désireuse d'entamer une relation sexuelle avec un homme plus âgé. Le lien avec le roman de Nabokov est souvent rompu dans cette vision contemporaine de la nymphette et toute trace d'abus et de violence envers elle semble effacée, ou du moins passée au second plan. Pourtant, paradoxalement, c'est cette perversion qui a propulsé le roman sur le devant de la scène en en faisant ce que beaucoup nomment une « cause célèbre ».

2 Dans cet article, il ne s'agit nullement de revenir sur la grande qualité du roman Lolita sur un plan esthétique mais de mettre en lumière la manière dont il a été reçu, ou plutôt rejeté, et ce pour des raisons morales, par différentes institutions gouvernementales et judiciaires. Par bien des aspects, la réception de Lolita et sa censure sont représentatives des difficultés que nombre de grands auteurs rencontrèrent au $20^{\mathrm{ème}}$ siècle pour faire publier leurs ouvrages en langue anglaise dans les pays anglo-saxons. Ainsi, pour plusieurs d'entre eux, Paris fut la ville où leurs livres, acclamés aujourd'hui mais décriés à l'époque pour leur obscénité supposée, purent enfin voir le jour: Nabokov dut se résoudre à publier en France en passant par la maison Olympia Press, tout comme avant lui Henry Miller (chez Obelisk Press) ou James Joyce $^{1}$ (chez Shakespeare and Company); D. H. Lawrence, lui, publia L'Amant de Lady Chatterley à Florence.

3 Pour donner un aperçu des déboires judiciaires que le roman Lolita a connus, je m'appuierai sur la correspondance de Nabokov mais surtout sur les archives Gallimard. Celles-ci, qui n'ont jamais été publiées, m’ont été rendues accessibles par M. Éric Legendre, des éditions Gallimard, et ce grâce au Professeur Maurice Couturier, chercheur et traducteur de Nabokov, notamment du roman Lolita, chez Gallimard. Mais 
cet article met également en perspective les différents types de censure dont a souffert Nabokov, notamment durant la première moitié de sa carrière, où il écrivait en russe.

\section{Nabokov et la censure en Russie}

4 Avant d'être l'écrivain américain ayant donné le jour à Lolita, Vladimir Nabokov avait publié neuf romans en russe, ainsi que des poèmes et des nouvelles, entre autres. Aucun de ces écrits ne traitait de l'amour d'un homme d'âge mûr pour une jeune adolescente ${ }^{2}$, et pourtant tous furent soumis à la censure ${ }^{3}$. En effet, l'œuvre russe de Nabokov était interdite en URSS, mais cela était dû aux raisons politiques que l'on connaît: les Nabokov étaient ce que l'on appelle des «Russes Blancs » et le père de Nabokov avait appartenu au gouvernement provisoire renversé par les Bolchéviques en 1917, ce qui en faisait un opposant politique au régime soviétique.

5 Si les écrits en langue russe de Nabokov étaient interdits en URSS, ils étaient diffusés en Europe, notamment auprès des émigrés russes. Or, là aussi, Nabokov vit son art partiellement muselé pour des raisons politiques. Ainsi, le chapitre 4 de son dernier roman russe, Le Don, ne fut pas publié par Sovremennye Zapiski car, comme l'écrit Maurice Couturier, "la représentation de Tchernychevski fournie par ce texte ne correspondait absolument pas à celle qui faisait loi parmi les émigrés russes » (Couturier 1979, 25).

6 Si ce chapitre fut censuré, c'est qu'il contenait une biographie peu flatteuse de Nikolaï Tchernychevski, écrite par le narrateur du Don, Fiodor Godounov-Tcherdyntsev. Or Tchernychevski fut une grande source d'inspiration pour l'esthétique soviétique puisqu'il codifia durablement l'esthétique réaliste russe en mettant l'art au service du réel; les Soviétiques, eux, le mirent au service du Marxisme ${ }^{4}$. L'idéologie de Tchernychevski est notamment exposée dans son roman Que faire? (publié en 1863) et l'admiration de Lénine pour ses idées était telle qu'il intitula son propre traité révolutionnaire (publié en 1902) de la même manière, Que faire? Paradoxalement, même si Sovremennye Zapiski était une revue littéraire assez libérale, et non affiliée à l'URSS, ses éditeurs refusaient d'écorner une figure majeure comme Tchernychevski. Dans son introduction au Don, Nabokov souligna bien l'ironie inhérente au refus de la revue Sovremennye Zapiski de publier son chapitre quatre :

La principale revue d'émigrés Sovrémennye zapiski, publiée à Paris par un groupe d'anciens membres du Parti social révolutionnaire, fit paraître le roman en feuilletons ( $\mathrm{n}^{\text {os }}$ 63-67, 1937-1938), en omettant cependant le chapitre quatre qui fut écarté pour les mêmes raisons qui poussent Vassiliev, dans le chapitre trois (p. 219-220), à le rejeter, et cela à cause de la biographie qu'il contient : un assez bel exemple de la vie se trouvant dans l'obligation d'imiter l'art même qu'elle condamne. (Nabokov 2010, 3)

7 Dans l'extrait du chapitre trois en question, l'éditeur Vassiliev souligne que c'est la dimension antisociale de cette biographie qui le pousse à rejeter le manuscrit de Fiodor. C'est donc une raison politique qui motive ce refus et, de l'aveu de l'éditeur, aucune considération esthétique n'est pertinente :

"Voici votre manuscrit", dit soudain Vassiliev, fronçant les sourcils et lui remettant la chemise. 'Prenez-le. Il ne saurait être question que je participe à sa publication. Je supposais que c'était un travail sérieux, et il se trouve que ce n'est qu'une improvisation téméraire, antisociale et malveillante. [...] Il y a certaines traditions de la vie publique russe qu'un écrivain honorable ne se permettrait jamais de tourner 
en ridicule. Il m'est absolument indifférent que vous ayez du talent ou pas : tout ce que je sais, c'est que brocarder un homme dont les œuvres et les souffrances ont été une nourriture pour des millions d'intellectuels russes est indigne de tout talent.' (Nabokov 1937, 219-220; c'est moi qui souligne)

9

Cependant, les deux types de censure qu'a connus Nabokov ne peuvent totalement se diviser le long de la démarcation linguistique de sa carrière. D'une part, Lolita fut également interdit en URSS 5 (qu'il s'agisse de la version autotraduite par Nabokov en 1967, d'autres traductions introduites illégalement par des éditions samizdat, ou de la version en anglais) : s'il est assuré que le roman fut interdit en raison des origines de Nabokov, et donc pour des raisons politiques qui s'appliquaient à l'intégralité de son œuvre, d'autres sources mentionnent que le roman fut interdit en raison de sa pornographie supposée. D'autre part, c'est en russe que Nabokov aborda pour la première fois la question taboue de la pédophilie, dans sa nouvelle "L'Enchanteur » (Nabokov 1986). Nabokov n'eut cependant pas à faire face à des questions morales pour cette œuvre première puisqu'elle ne fut publiée qu'après sa mort. Nabokov reconnut bien volontiers le rôle de précurseur que cette nouvelle russe avait pour son chef d'œuvre américain. Dans sa postface au roman, «On a book entitled Lolita », il l'évoquait en ces termes :

a prototype of my present novel, a short story some thirty pages long. I wrote it in Russian, the language in which I had been writing novels since 1924 (the best of these are not translated into English, and all are prohibited for political reasons in Russia). (Nabokov 1955, 293 ; c'est moi qui souligne)

L'objectif de cette postface était de nier l'immoralité de Lolita, et donc de la prémunir de la censure. Or il est intéressant de remarquer que, dès la première page, Nabokov informe son lectorat anglophone qu'il a déjà été victime de la censure politique en russe. En pleine guerre froide, l'intention était peut-être de suggérer une similitude de méthodes peu flatteuse entre l'URSS et les milieux intellectuels de l'Ouest.

Car avant d'être soumis à la censure par des gouvernements occidentaux, Lolita a été victime d'un autre type de censure, celle des éditeurs.

\section{Lolita, ou le combat contre la censure}

\section{La recherche d'un éditeur aux Etats-Unis}

Lorsque Nabokov décide de publier Lolita, il est professeur à la prestigieuse université de Cornell aux Etats-Unis : cet emploi sort enfin sa famille d'une précarité qui a duré une grande partie de leur exil et, surtout, il donne à Nabokov une stabilité qui lui permet de se consacrer à ses deux passions, l'entomologie et la littérature. Le sujet de Lolita l'incite donc à approcher les maisons d'édition avec une demande expresse : ne publier le roman que sous un pseudonyme afin de ne pas embarrasser son université et de ne pas perdre cet emploi. Mais, comme il le souligne dans la postface de son roman, le sujet révulse les éditeurs éventuels :

The four American publishers, W, X, Y, Z, who in turn were offered the typescript and had their readers glance at it, were shocked by Lolita to a degree that even my wary old friend F.P. had not expected. (Nabokov 1955, 294) 
Le refus de publier Lolita s'explique donc par le fait que les éditeurs jugeaient immoral le sujet du roman ${ }^{6}$. Comme le souligne John de St Jorre dans son livre Venus Bound: The Erotic Voyage of the Olympia Press and its Writers, il s'agissait plus précisément d'une autocensure de la part des éditeurs :

This was Senator McCarthy's America, where crossing the frontiers of convention in any literary or artistic endeavor could easily be equated with communist subversion and the practitioner hounded into oblivion. Moreover, American publishing in the mid-1950s was still in the dark ages, constrained by legal sanctions without and self-censorship within. (De St Jorre, 120)

Et c'est sans nul doute un genre d'autocensure similaire qui poussa Nabokov à proposer son livre sous un nom d'emprunt, tout autant que sa lucidité, quant à ce que beaucoup percevraient dans Lolita: une perversion, non une œuvre d'art. Et selon Edward de Grazia, avocat spécialiste du premier amendement américain ${ }^{7}$, c'est ce désir d'anonymat qui compliqua la publication américaine du roman :

Publication of Lolita under a pseudonym in the United Stated in 1954 would certainly have made difficult any legal defense of the novel. There would have been no apparent way for the publishers' lawyers to present evidence of the author's literary distinction or reputation, a matter crucial to successful defense. (De Grazia, 247)

15 En effet, dissimuler le nom de Nabokov signifiait qu'il était impossible de faire référence à ses œuvres antérieures, et donc de démontrer que l'auteur de Lolita était un artiste depuis longtemps, reconnu qui plus est, et non un pornographe à la recherche d'un succès de scandale. Par ailleurs, si Nabokov refusait d'assumer la paternité du roman, l'accusation aurait pu arguer que cela signifiait qu'il savait qu'il y avait quelque chose de douteux et répréhensible dans ce livre. De plus, de Grazia souligne que ce n'est pas nécessairement la peur de la censure qui guidait les éditeurs, mais la crainte des coûteux procès qui en découleraient :

Nabokov's Lolita could, I believe, have been successfully defended against charges of obscenity if a reputable publisher had brought it out in the mid-fifties. But the predictable legal expenses of a long legal battle to defend the novel were such that only a large and stable house, or a bold one, could have expected to publish Lolita successfully at that time. (De Grazia, 251)

Loin d'être stable ou même dotée d'une bonne réputation, c'est finalement la maison d'édition The Olympia Press, basée à Paris et dirigée par l'audacieux Maurice Girodias ${ }^{8}$, qui accepta de publier le roman.

\section{L'Affaire Lolita en France ${ }^{9}$}

Girodias était le fils de Jack Kahane, fondateur de The Obelisk Press, qui publia notamment Henry Miller (Tropic of Cancer), Lawrence Durrell (The Black Book) et Anaïs Nin (The Winter of Artifice). Dans les années 1950 et 1960, trois hommes combattaient la censure littéraire dans le monde anglo-saxon: Barney Rosset aux Etats-Unis, John Calder au Royaume-Uni, et Maurice Girodias, qui publiait à Paris des ouvrages en langue anglaise. Ce dernier était connu à la fois pour la publication de littérature érotique mais aussi pour avoir accepté des auteurs dont personne d'autre ne voulait; l'un n'allait pas sans l'autre puisque les revenus venant des ouvrages érotiques servaient souvent à financer des livres plus littéraires d'écrivains tels que Miller, Georges Bataille, Jean Genet ou Apollinaire. 
odias publia Lolita en septembre 1955 mais le roman passa presque inaperçu jusqu'à ce que l'écrivain britannique Graham Greene déclare, dans le Sunday Times de Noël 1955, qu'il s'agissait d'un des trois meilleurs romans qu'il avait lus cette année-là. En réponse, fin janvier 1956, le rédacteur en chef du Sunday Express, John Gordon, dénonça Lolita en ses termes: "the filthiest book I have ever read. Sheer unrestrained pornography. " (De Grazia, 259) D'autres personnalités de la scène littéraire ou des journalistes prirent parti pour ou contre le roman ${ }^{10}$, et le Home Office (ministère de l'Intérieur britannique) aurait fait pression sur le ministère de l'Intérieur français : la Brigade Mondaine rendit visite à Girodias dans ses bureaux d'Olympia Press en 1956 et demanda à prendre vingt-cinq livres pour les consulter, dont Lolita. Le 25 décembre de cette même année, un arrêté signé du ministre de l'Intérieur Jean Gilbert-Jules tomba : ces vingt-cinq livres étaient interdits à la vente et à la publication, et ce, en vertu de l'article 14 de la loi du 29 juillet 1881 sur la liberté de la presse. Or, comme Girodias le souligne dans son introduction à son édition de 1959 de Lolita (Nabokov 1959, 6), cette loi ne peut normalement porter que sur les écrits politiques; par ailleurs, elle ne concerne que la presse, comme son nom l'indique, et non les livres, comme le rappelle Couturier $(2011,123)$.

19 Girodias décida d'intenter un procès au gouvernement français et publia en avril 1957 un pamphlet, L'Affaire Lolita - Défense de l'écrivain. Nabokov refusa de prendre part au pamphlet ou au procès, car il ne souhaitait pas se mêler de la « lolitigation », ainsi qu'il l'appelle dans une lettre à Girodias en mars 1957 (Nabokov 1991, 210). Comme Nabokov l'explique à Jason Epstein, rédacteur en chef de Random House, dans un courrier en date du 20 février 1957, défendre en France la publication de son roman en anglais n'est pas essentiel pour lui puisque sa publication en français y est assurée :

I wish, of course, to give every possible support to Olympia, though personally I do not care if the ban will be lifted or not, since Gallimard is going to publish the French translation anyway. (Nabokov 1991, 203)

Girodias remporta son procès contre le gouvernement puisque, le 14 janvier 1958, le tribunal administratif annula l'interdiction prononcée par le ministère de l'Intérieur. Cependant, avec l'arrivée au pouvoir de De Gaulle en mai 1958 et les difficultés présentées par la guerre d'Algérie, le pouvoir de l'Etat se renforça et, lorsque le ministère de l'Intérieur fit appel de la décision du tribunal administratif, le conseil d'Etat confirma l'interdiction des vingt-cinq livres, dont Lolita, dans une décision en date du 17 décembre 1958. Cette fois-ci, le texte de loi invoqué était l'article 14 de la loi du 16 juillet 1949 sur les publications destinées à la jeunesse.

21 A partir de fin décembre 1958, donc, le roman Lolita publié par Olympia Press était de nouveau interdit. Or, en avril 1959, la version française du roman, traduite par Eric Kahane (le frère de Girodias) parut, mais elle n'était, elle, pas soumise à cette interdiction. Girodias s'appuya sur cette contradiction pour intenter un nouveau procès contre le gouvernement; il demanda des dommages et intérêts, cette fois en arguant que le principe d'égalité des citoyens avait été violé. Le 21 juillet 1959, après que Girodias eut accepté de retirer sa plainte, le ministre de l'Intérieur leva l'interdiction portant sur Lolita, mais pas sur les vingt-quatre autres ouvrages. 


\section{La censure de Lolita dans le reste du monde (ou presque)}

nombreux autres pays ont opposé résistance au roman : le 22 juillet 1959 , Cla Gallimard fut informé que des exemplaires de Lolita avaient été saisis dans trois librairies de la région de Constantine en Algérie (archives Gallimard); la Belgique interdit le roman entre le 15 février 1960 et le 28 mars de la même année ${ }^{11}$ et, dans sa lettre ouverte en date du 11 mars 1960 adressée au ministre de l'Intérieur belge, M. René Lefebvre, Girodias mentionne que le seul pays où le roman est encore interdit (à part la Belgique donc) serait la Birmanie, puisque la censure en Argentine a finalement été levée (archives Gallimard, Girodias, 11 mars 1960). Les pays anglo-saxons ne furent pas en reste puisque l'Australie interdit le roman en $1955^{12}$, le Canada en 1958, et la Nouvelle-Zélande en 1960. En date du 13 novembre 1956, Nabokov informa Jason Epstein que Lolita aurait été interdite aux Etats-Unis et au Royaume Uni en lui transmettant une coupure de presse stipulant :

\section{OLYMPIA PRESS BANNED TITLES}

Practically all English titles printed by Olympia Press in Paris (sorry, we cannot give the address) have been banned in England and America. Latest to be banned by U.S. Customs is 'Lolita', a new novel by Vladimir Nabokov, which was called 'one of the best novels in 1955' by Graham Greene in The Sunday Times. (Nabokov 1991, 193)

Mais les choses ne sont pas tout à fait claires, pour le Royaume-Uni par exemple. En effet, si l'on en croit les annotations de Dmitri Nabokov sur les lettres de son père Vladimir, le roman ne fut pas interdit (Nabokov 1991, 198) ; c'est également ce qu'écrit John Calder dans The Garden of Eros: The Story of the Paris Expatriates and the Post-War Literary Scene: «British Customs, surprisingly, cleared the book.» (Calder, 198) Mais, selon d'autres sources, le Home Office ordonna aux douanes britanniques de saisir tout exemplaire entrant au Royaume-Uni (Capon et Scott), ce qui dura jusqu'en 1957; Graham Greene écrivit d'ailleurs à Nabokov en janvier 1957 :

I thought Lolita a superb book + I am now, as a director of a publishing firm in England, trying to arrange its publication. In England, one may go to prison, but there couldn't be a better cause! (Nabokov 1991, 198)

Cependant, ce n'est qu'en 1959 que Lolita parut au Royaume-Uni chez Weidenfield \& Nicholson ${ }^{13}$ : en effet, les éditeurs attendaient la promulgation d'une loi, the Obscene Publications Act (De Grazia, 261; De St Jorre, 147-148), comme George Weidenfield l'expliqua à Nabokov le 28 janvier 1959 :

we should not publish before this new bill has become effective. [...] Under the new bill not only will literary merit be taken into account in deciding the fate of the book but the defence will be able to call witnesses to testify to the book's merits. (Nabokov 1991, 279)

Aux Etats-Unis, il n'y eut jamais de procès, même si certaines bibliothèques interdirent la présence de Lolita sur leurs étagères (De Grazia, 269). Par contre, pour ce qui est des douanes américaines, elles saisirent bien quelques ouvrages en 1956 mais ne donnèrent pas suite (Nabokov 1991, 194), ce qui rassura Girodias comme Nabokov quant à une publication américaine. Le 23 mars 1957, Nabokov écrivit à Ivan Obolenski pour le remercier de son intérêt pour publier Lolita mais qu'il préférait attendre les résultats du procès français ainsi que l'opinion de la Cour Suprême qui statuait à ce moment-là sur la question de l'obscénité (Nabokov 1991, 211-212). Le 24 juin 1957, le verdict de la cour suprême fut rendu dans la décision Roth versus United States; elle allait par la suite être convoquée pour protéger toute production ayant la moindre importance ou valeur, 
qu'elle soit littéraire, artistique ou sociale; c'était donc une décision majeure dans la lutte contre la censure :

During the next several decades American artists and writers and their publishers, producers, exhibitors, distributors, and managers would base their struggle to gain freedom from censorship upon the premise established in Roth that artistic expression was meant to be protected as fully by the First Amendment as were religious and political expression. (De Grazia, 321)

Quand cette décision de justice fut rendue, Nabokov exprima sa déception car elle n'avait pas statué clairement sur ce qu'était ou non l'obscénité, et il douta qu'elle puisse faire quoi que ce soit pour Lolita (Nabokov 1991, 222). Cependant, cette décision rendit possible une version américaine du roman, qui parut l'année suivante, en 1958, chez Putnam.

\section{Censure et valeur littéraire}

C'est cette question de valeur, artistique notamment, qui est évoquée dans la loi britannique de 1959, The Obscene Publications Act ${ }^{14}$, tandis que Roth versus United States affirme plutôt que les seules œuvres qui peuvent être interdites, et donc non protégées par le premier amendement, sont celles n'ayant pas la moindre valeur sociale pouvant les racheter :

All ideas having even the slightest redeeming social importance [...] have the full protection of the guaranties, unless excludable because they encroach upon the limited area of more important interests. But implicit in the history of the First Amendment is the rejection of obscenity as utterly without redeeming social importance ${ }^{15}$.

C'est cet argument de valeur esthétique que Nabokov utilisa pour ne pas se joindre à Girodias dans son procès contre le gouvernement :

My moral defense of the book is the book itself. I do not feel under any obligation to do more. [...] On the ethical plane, it is of supreme indifference to me what opinion French, British or any other courts, magistrate or philistine readers in general, may have of my book ${ }^{16}$. (Nabokov 1991, 210)

Il insista également en plusieurs endroits sur la distinction entre littérature et obscénité :

I know that LOLITA is my best book so far. I calmly lean on my conviction that it is a serious work of art, and that no court could prove it to be 'lewd and libertine'. All categories grade, of course, into one another: a comedy of manners written by a fine poet may have its 'lewd' side; but LOLITA is a tragedy. 'Pornography' is not an image plucked out of context; pornography is an attitude and an intention. The tragic and the obscene exclude each other. (Nabokov 1991, 194)

Il reconnaissait cependant avoir abordé l'un des trois sujets tabous de l'époque et effrayé ainsi les éditeurs potentiels :

Their refusal to buy the book was based not on my treatment of the theme but on the theme itself, for there are at least three themes which are utterly taboo as far as most American publishers are concerned. The two others are: a Negro-White marriage which is a complete and glorious success resulting in lots of children and grandchildren; and the total atheist who lives a happy and useful life, and dies in his sleep at the age of 106. (Nabokov 1955, 295)

31 Nabokov souligna souvent à quel point il ne voulait pas d'un succès de scandale, et que c'était bien la qualité littéraire du roman qui primait à ses yeux, notamment quand il 
écrivait dans son essai « On a book entitled Lolita » : « Lolita was the record of my love affair with the [...] English language. » (Nabokov 1955, 298) Or il est certains critiques qui virent dans les qualités du roman une raison supplémentaire de décrier le roman. Ce fut le cas pour Orville Prescott du New York Times:

'Lolita' is not crudely crammed with Anglo-Saxon nouns and verbs and explicitly described scenes of sexual violence. Its depravity is more refined. Mr. Nabokov, whose English vocabulary would astound the editors of the Oxford Dictionary, does not write cheap pornography. He writes highbrow pornography (Prescott).

Si beaucoup ont été choqués par le sujet tabou de Lolita, c'est son écriture qui devrait secouer, bouleverser les lecteurs. L'écrivain Léonid Guirchovitch décrit justement sa redécouverte de la langue russe de Nabokov dans son autotraduction de Lolita en évoquant les tabous et interdits littéraires que l'écrivain bilingue avait su briser :

Ce ne sont pas les idées de Nabokov qui ont influencé les jeunes écrivains mais son style. [...] Il bousculait tous les tabous avec brio. Ma première lecture a été bouleversante du fait de cette liberté avec laquelle Nabokov a mis en pièces tous les interdits qui pesaient sur moi. (Guirchovitch, 89)

\section{Conclusion : Lolita et la censure, 60 ans après}

Lolita a désormais soixante ans, mais régulièrement, des bibliothèques aux Etats-Unis font face à des demandes de retirer le livre de leurs étagères (Marsh) ou des enseignants sont remis en question pour l'avoir mis au programme en littérature américain $\mathrm{e}^{17}$. L'exemple le plus récent du fait que la question de la censure de Lolita n'a pas vieilli, soixante ans après sa publication, se trouve en Russie.

En 2011, un représentant officiel de l'église orthodoxe de Russie, Vsevolod Chaplin, a appelé les autorités à interdire Lolita de Nabokov et Cent ans de solitude de Gabriel Garcia Marquez dans les établissements d'enseignement secondaire car ces romans « justifiaient la pédophilie» (Associated Press, ma traduction). En 2013, durant la représentation d'une adaptation du roman à Saint-Pétersbourg, le metteur en scène a été roué de coups et traité de pédophile, tandis que le musée Nabokov dans cette même ville et la maison d'enfance de Nabokov dans la campagne pétersbourgeoise se sont vus couverts de graffiti portant la même insulte (Idov). Toujours en 2013, les procureurs de la région de Stavropol ont exigé que les écoles locales se débarrassent de leurs exemplaires de Lolita et d'autres ouvrages, russes comme étrangers, car ceux-ci n'étaient pas compatibles avec la mission d'éducation de ces écoles, ils effrayaient les enfants et encourageaient le crime (Krainova). Cette démarche est possible en Russie en raison de la loi fédérale réprimant la "propagande de l'homosexualité et de la pédophilie auprès des mineurs » qui est entrée en vigueur le 17 mars 2012 à SaintPétersbourg ${ }^{18}$.

De même qu'en décembre 1958, la deuxième interdiction française touchant Lolita s'appuyait sur la loi du 16 juillet 1949 sur les publications destinées à la jeunesse, la censure russe prétend protéger les enfants d'un livre qui n'est nullement affiché comme étant destiné à un public mineur. Force est de constater que ces décisions de justice tendent à confirmer l'adage selon lequel, malheureusement, l'histoire se répète. 


\section{BIBLIOGRAPHIE}

Alladaye, René. « Le Cas Lolita », Cycnos 9 (2008). 21 juillet 2017.

http://revel.unice.fr/cycnos/index.html?id=1174

Associated Press. « Russian church urges ban on Lolita ». In CBC News, 28 septembre 2011. 23

septembre 2015.

<http://www.cbc.ca/news/arts/russian-church-urges-ban-on-lolita-1.1039214>

Boyd, Brian. Vladimir Nabokov: the American Years. Princeton: Princeton University Press, 1991.

Calder, John. The Garden of Eros: The Story of the Paris Expatriates and the Post-War Literary Scene. 2013. Richmond: Alma Books, 2014.

Capon, Felicity et Scott, Catherine. « Top 20 books they tried to ban ». In Telegraph, (20 octobre 2014). 21 juillet 2017.

<http://www.telegraph.co.uk/culture/books/booknews/9900733/Top-20-books-they-tried-toban.html>

Couturier, Maurice. Nabokov ou la Tentation française. Paris : Gallimard, 2011.

---. Vladimir Nabokov. Lausanne : L'Age d'Homme, 1979.

De Grazia, Edward. Girls Lean Back Everywhere: The Law of Obscenity and the Assault on Genius. New York: Random House, 1992.

De St Jorre, John. Venus Bound: The Erotic Voyage of the Olympia Press and its Writers. New York : Random House, 1994.

Edel-Roy, Agnès. « Lolita, ou 'l'ombre d'une branche russe'. Etude de l'auto-traduction », Miranda [Online] 3 (2010). 21 juillet 2017.

<http://miranda.revues.org/1536>

« Editeurs, les lois du métier ». Exposition numérique. 21 juillet 2017. <http://

editeurslesloisdumetier.bpi.fr/bpi_loi-edition/fr/chronologie.html\#div_0>

Girodias, Maurice. Lettre ouverte à René Lefebvre, 11 mars 1960, Paris. Paris : Archives Gallimard.

Godin, Huguette. Lettre à Gaston Gallimard, 23 décembre 1956, Paris. Paris: Archives Gallimard.

Girodias, Maurice. « Publisher's Digression ». In Vladimir Nabokov. Lolita. 1955. Paris: Olympia

Press, 1959.

Guirchovitch, Léonid. « Vladimir Nabokov ». Transfuge 6 (mars 2005) : 88-89.

Idov, Michael. «The Turn Against Nabokov ». In The New Yorker, 27 février 2013. 21 juillet 2017.

<http://www.newyorker.com/books/page-turner/the-turn-against-nabokov>

Krainova, Natalya. «Stavropol Prosecutors Seek to Ban Nabokov in Schools ». In The Moscow

Times, 22 novembre 2013. 21 juillet 2017.

<http://www.themoscowtimes.com/news/article/stavropol-prosecutors-seek-to-ban-nabokovin-schools/490036.html>

Lay, Amy. « Lolita ». In « Banned » Exposition numérique, 9 octobre 2013. 21 juillet 2017. <http:// blog.naa.gov.au/banned/2013/10/09/lolita/> 
Marsh, Kathy. « 'Lolita' could be pulled from shelves ». In Wesh.com, 24 janvier 2006. 21 juillet 2017.

<http://www.wesh.com/-Lolita-Could-Be-Pulled-From-Library-s-Shelves/13104236>

Nabokov, Dmitri et al. (ed.). Vladimir Nabokov, Selected Letters, 1940-1977. 1989. Londres : Vintage, 1991.

Nabokov, Vladimir. «L'Enchanteur ». 1986. Trad. Gilles Barbedette. In Vladimir Nabokov. Cuvres romanesques complètes. Ed. Maurice Couturier, trad. Maurice Couturier et al. vol. ii. Paris :

Gallimard, 2010. 551-601.

---. Le Don. 1937. In Vladimir Nabokov. Euvres romanesques complètes. Ed. Maurice Couturier, trad. Maurice Couturier et al. vol. ii. Paris: Gallimard, 2010.

---. Lectures on Literature. Ed. Fredson Bowers. San Diego: Harcourt, 1982.

---. « On a book entitled Lolita ». In Vladimir Nabokov. Lolita. 1955. In Vladimir Nabokov, Novels, 1955-1962. Ed. Brian Boyd. New York: Library of America, 1996. 1-298.

---. « Russians Writers, Censors and Readers ». In Lectures on Russian Literature. Ed. Fredson Bowers. New York: Harcourt, 1981. 1-12.

Prescott, Orville. « Books of the Times ». In New York Times, 18 août 1958: 27.

Roscoff, Nadia. « On Literary (Ab)normality: Lolita and Self-Translation ». Université d'Alberta, 2015. 21 juillet 2017.

<https://era.library.ualberta.ca/downloads/gh93h213x>

\section{NOTES}

1. Dans la préface à Lolita, il est fait mention de la décision de justice qui rendit possible la publication de Ulysses de Joyce en 1933 : «see in this respect the monumental decision rendered December 6, 1933, by Hon. John M. Woolsey, in regard to another, considerably more outspoken, book. » (Nabokov 1955, 4)

2. La nouvelle "L'Enchanteur », qui traite de ce sujet et dont il est question plus loin, ne fut pas publiée du vivant de Nabokov.

3. Sur la censure en Russie et en URSS, lire « Russians Writers, Censors and Readers " (Nabokov 1981). Nabokov insistait souvent sur le fait que la censure sous les Tsars était moins forte et pernicieuse que pendant l'URSS, et soulignait la relative liberté dont disposaient les écrivains russes juste avant la Révolution: "The system of censorship that he [Tsar Nicholas I] evolved lasted till the 1860s, was eased by the great reforms of the sixties, stiffened again in the last decades of the century, broke down for a short spell in the first decade of this century, and then had a most sensational and formidable comeback after the Revolution under the Soviets. " (Nabokov 1981, 3)

4. Je remercie Agnès Edel-Roy pour ses explications sur l'esthétique et la politique russes.

5. Sur la réception de Lolita en URSS, puis dans la Fédération de Russie, lire Edel-Roy (2010) et Roscoff (2015), 193-216.

6. Dans sa postface, Nabokov fait référence à ce qu'il nomme «this idiotic accusation of immorality » (Nabokov 1955, 296).

7. Il défendit notamment la publication de Tropic of Cancer de Miller et de Naked Lunch de Burroughs; sa victoire au tribunal en 1966 pour le procès Burroughs mit fin à la censure littéraire aux Etats-Unis. 
8. Pour un portrait de l'éditeur Maurice Girodias et une chronologie des lois sur l'édition en France, voir la version numérique de l'exposition de la BPI « Editeurs les lois du métier » : http:// editeurslesloisdumetier.bpi.fr/bpi_loi-edition/fr/index.html 9. Sur ce sujet, lire aussi : Couturier (2011), 110-134; Boyd (1991), 299-301 et Alladaye (2008). 10. Ainsi, Huguette Godin, chargée du courrier littéraire international à l'AFP, exprime la double tension dans laquelle la promotion de Lolita plaçait tout journaliste ou maison d'édition : «J'ai lu 'Lolita' : je pense que c'est évidemment un livre 'impossible' dans l'état actuel de la législation et des mœurs. Je pense aussi que c'est un livre remarquable, à plus d'un point de vue. » (Godin, 23 décembre 1956)

11. Le 20 avril 1960, la maison Gallimard fut informée par un certain D. Pichon (archives Gallimard), du département étranger Hachette à Bruxelles, que l'interdiction de la vente de Lolita en Belgique venait d'être levée et incluait un extrait du Moniteur (l'équivalent belge du Bulletin officiel français) relatif à l'affaire :

«Considérant que la circonstance d'un complément d'enquête justifie de lever en ce moment l'interdiction de la vente, de l'exposition en vente, de l'introduction et de la distribution de la publication étrangère de Lolita (Wladimir Nabohof [sic]) ;

Sur la proposition de Notre Ministre de l'Intérieur,

Nous avons arrêté et arrêtons :

Article $1^{\text {er }}$ : Les dispositions de l'article $1^{\text {er }}$ de Notre arrêté du 15 février 1960 sont rapportées en ce qu'elles concernent l'interdiction de l'introduction en Belgique en vue de commerce ou de la distribution, de la vente, de l'exposition en vente et de la distribution de la publication étrangère de Lolita (Wladimir Nabohof [sic]).

Art. 2 : Notre Ministre de l'Intérieur est chargé de l'exécution du présent arrêté.

Donné à Bruxelles, le 28 mars 1960. »

12. Certains textes de loi relatifs à l'interdiction en Australie sont reproduits sur le blog que les archives nationales australiennes ont créé à l'occasion d'une exposition sur la censure en Australie, notamment à l'entrée dédiée à Lolita: http://blog.naa.gov.au/banned/2013/10/09/ lolita/

13. John Calder avait obtenu de Girodias les droits pour Lolita mais Nabokov s'y opposa : « [a trial] had gained me the reputation in some circles of being soft on communism [...]. Vladimir Nabokov, like nearly all émigrés from Russia and Eastern Europe, was very right-wing and opposed to liberalism of any kind. He made it clear to Girodias that he did not want me to be his British publisher.» (Calder, 151-2)

14. Voir l'intégralité de la loi à cette adresse: http://www.legislation.gov.uk/ukpga/1959/66/ pdfs/ukpga_19590066_en.pdf

15. Voir l'intégralité de la décision de la cour suprême à cette adresse: https:// supreme.justia.com/cases/federal/us/354/476/case.html

16. Notons que Nabokov avait déjà réfuté la prise en compte d'arguments moraux pour estimer la valeur esthétique d'une œuvre quand il était professeur de littérature. Ainsi, dans son cours sur Madame Bovary, il évoquait en ces termes le procès contre Gustave Flaubert : "Indeed, the novel was actually tried in a court of justice for obscenity. As if the work of the artist could ever be obscene. That was exactly a hundred years ago. In our days, our times... But let me keep to my subject. » Ce sujet était pour lui tellement trivial que ces quelques mots sont les seuls qu'il consacre au procès contre Flaubert dans son cours (Nabokov 1982, 125).

17. Voir par exemple la page de l'exposition numérique «Lolita " évoquant le débat soulevé quand Lolita fut proposé au programme de littérature américaine de l'université nationale australienne : http://blog.naa.gov.au/banned/2013/10/09/lolita/

18. Notons que Milonov, l'auteur de cette loi, a reçu le 14 septembre 2015 une décoration de la part du président Vladimir Poutine pour son travail consciencieux... 


\section{RÉSUMÉS}

Cet article met en lumière la tension entre art et politique dans la carrière de Nabokov. Avant de devenir un auteur américain, Nabokov a souffert de censure politique dans son pays d'origine, sans que les qualités littéraires de son œuvre ne soient prises en compte. Puis, cet article étudie la manière dont le roman Lolita a été reçu, ou plutôt rejeté, par différentes institutions gouvernementales et judiciaires, en France et dans le monde. Ce rejet n'était pas motivé par des raisons esthétiques mais morales, et par bien des aspects, la censure de Lolita est représentative des difficultés que de grands auteurs tels que Nabokov, James Joyce, D. H. Lawrence ou Henry Miller ont rencontrées au $20^{\text {ème }}$ siècle pour faire publier leurs ouvrages. La correspondance de Nabokov et des extraits d'archives Gallimard inédites donneront un aperçu des déboires judiciaires que le roman a connus. Finalement, les qualités esthétiques des œuvres littéraires furent prises en compte par les institutions judiciaires, ce qui permit de lever toute censure à l'encontre de Lolita.

This article sheds light on the tension between art and politics in Nabokov's career. Before he became an American author, Nabokov was subjected to political censorship in his motherland, with a complete disregard of and lack of interest in the literary qualities of his novels. Then, the article studies how Lolita was banned in different countries, be it in France or elsewhere in the world. The reason for this ban was not aesthetics but ethics. In many respects, the censorship Lolita suffered from is an illustration of the difficulties that great authors such as Nabokov, James Joyce, D. H. Lawrence or Henry Miller were subjected to when it came to publishing their novels in the $20^{\text {th }}$ century. Nabokov's letters and exclusive archives from Gallimard will show some of the judicial mishaps / streaks of bad luck that Nabokov's novel met with. In the end, literary quality was made part of judicial decisions and it is thanks to its aesthetic value that censorship on Lolita was finally lifted.

\section{INDEX}

Keywords : censorship, perversity, trials, Olympia Press

Mots-clés : censure, perversion, procès, Olympia Press

\section{AUTEURS}

\section{JULIE LOISON-CHARLES}

Maître de conférences

Université de Lille

julie.charles@univ-lille3.fr 